\title{
Presence of Concomitant Asymptomatic and Symptomatic Emboli RESULTING FROM ACUTE STAPHYLOCOCCUS AUREUS ENDOCARDITIS
}

\author{
Jason T. Bradley, MD, Nicole M. Orr, MD, and Siva Ramachandran, MD
}

\begin{abstract}
A 25-year-old male intravenous heroin user with Hepatitis C presented with a syncopal episode and left sided weakness. Examination revealed sub-conjunctival hemorrhages and diffuse bilateral petechiae on his lower extremities. He had multiple irregular, erythematous, painless macules on his hands and feet, and linear, red, subungal lesions bilaterally. These findings were consistent with Janeway lesions and splinter hemorrhages, respectively. His vital signs were stable on admission. Cardiac auscultation revealed a grade $2 / 6$ holosystolic apical murmur radiating to the axilla. A brain MRI revealed multiple areas of hyperdensity, particularly in the right parietal lobe (Figure 1). Blood tests revealed leukocytosis and six of six blood cultures yielded methicillin sensitive Staphylococcus Aureus. The patient was treated with intravenous nafcillin and gentamicin.
\end{abstract}

One week later the patient's neurologic status improved. A transesophageal echocardiogram was performed and revealed both a vegetation on the posterior leaflet of an otherwise normal mitral valve and moderate mitral regurgitation. The following day, the patient developed diarrhea that was positive for both heme and Clostridium difficile toxin. Metronidazole was started and an abdominal CT scan was ordered (Figure 2). Although there was no corresponding clinical presentation, imaging revealed bilateral renal infarcts, a splenic infarct, and sigmoid thickening. The patient regained full neurologic functioning within two weeks of initial presentation and was discharged after a 6 week course of intravenous nafcillin. He is currently awaiting a mitral valve replacement.

In the setting of presumed septic emboli to both the skin and brain secondary to bacterial endocarditis, we were concerned that the heme positive stools were the result of embolization to the mesenteric arteries. Although the abdominal CT showed no evidence of bowel infarction and sigmoid thickening is non-specific, the renal and splenic infarcts were determined to be secondary to septic emboli. A previous case of bacterial endocarditis with simultaneous multiple organ involvement including the kidney, spleen, brain, skin, and intestines has not been documented. This case demonstrates that in the setting of diffuse symptomatic septic emboli, involvement of additional organ systems is likely but may be missed due to absence of clinical findings.

Concomitant symptomatic emboli to more than one organ system are rare according to the literature. It is interesting to note that petechiae occur in only 20-40\%, Osler nodes in only $10-25 \%$ of patients, and Janeway lesions in less than $10 \%$ of patients with bacterial endocarditis. Splenic septic emboli in infective endocarditis are a common finding and incidental splenic infarcts were found in $38 \%$ of 29 asymptomatic patients in one study.

Majumdar et al. found that $18 \%$ of 354 patients with infective endocarditis had renal involvement and $45 \%$ of that subpopulation was found to have localized renal infarction, making it the most common renal lesion. The authors of that study acknowledge this renal subpopulation does not represent all patients with endocarditis but a prospective study to assess symptomatic and asymptomatic renal involvement has not yet been done.

The incidence of septic emboli to the brain has been well documented. Whether or not embolization is a result of vegetation size is currently

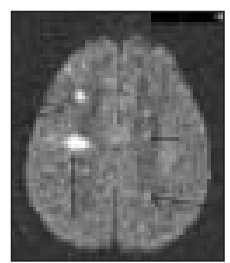

Figure 1. MRI of the brain identifying multiple areas of septic emboli.

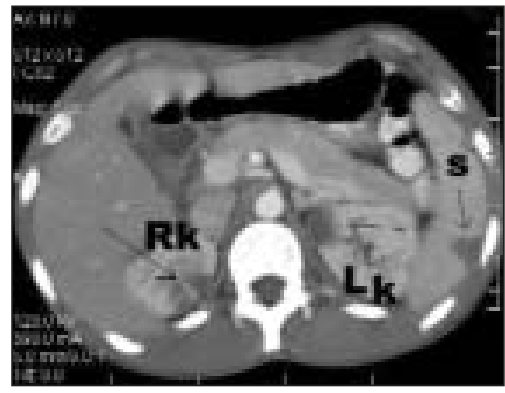

Figure 2. CT of the Abdomen identifying multiple embolic infarcts of the spleen $(S)$ and bilateral kidneys $(R k, L k)$.

disputed, but current data suggest that vegetations greater than one centimeter in length have increased risk for cerebral embolism ${ }^{4}$. Brain embolization has been previously described in a case report and several studies $^{5,6}$, but not with concomitant lesions to other organ systems. Eighteen percent of patients with mitral valve endocarditis develop stroke and in over one-half of these cases, stroke was the initial presenting symptom 5 . The authors concluded that all patients with endocarditis and neurologic symptoms should undergo neurologic imaging to determine the exact location of the lesions. Bakshi et al. even concluded that neuroimaging should be used to diagnose bacterial endocarditis in patients presenting with neurologic symptoms and nonspecific constitutional symptoms. In addition, that study cites that $50 \%$ of patients with neurologic sequelae of bacterial endocarditis do not have clinical evidence of additional peripheral emboli.

In conclusion, although the patient was admitted for symptomatic emboli to the skin and brain, non-symptomatic emboli to the kidney, spleen, and intestine were incidentally found. It is well known that infective endocarditis can infect the spleen, kidney, and brain, but normally such emboli are symptomatic and isolated. This case demonstrates that asymptomatic lesions may also be present in the face of already existing symptomatic emboli and careful investigation of critical organ systems should be done empirically.

The authors encountered this case during their time with the Department of Pulmonary and Critical Care at Hahnemann University Hospital, Philadelphia, PA.

\section{References}

1. Saccente, M. \& Cobbs, C.G. (1996). Clinical approach to infective endocarditis. Cardiology Clinics , 14 (3), 351-62

2. Ting, W., Silverman, N.A., Arzouman, D.A., \& Levitsky, S. (1990). Splenic septic emboli in endocarditis, Circulation, 82 (5),105-9

3. Majumdar,A., Chowdhary, S., Ferreira, M.A., Hammond, L.A., Howie, A.J., Lipkin, G.W. \& Littler, W.A. (2000). Renal pathologic findings in infective endocarditis. Nephrol Dial Transplant, 15 (11), 1782-7.

4. Krapf, H., Skalej, M., \& Voigt, K. (1999). Subarachnoid hemorrhage due to septic emboli infarction in infective endocarditis. Cerebrovascular Disease 9 (3), 182-4.

5. Sexton, D.J. \& Spelman, D. (2003). Current best practices and guidelines. Assessment and management of complications in infective endocarditis. Cardiology Clinics, 21 (2), 273-82.

6. Bakshi, R, Wright, P.D., Kinkel, P.R, Bates, V.E., Mechtler, L.L., Kamran, S. Pullicino, P.M, Sirotkin, I, \& Kinkel W.R. (1999). Cranial magnetic resonance imaging findings in bacterial endocarditis: the nueroimaging spectrum of septic brain embolization demonstrated in twelve patients. Journal of Neuroimaging, 9 (2), 78-84. 